\title{
A performance study of the suitability of adaptive boosting in red acne detection
}

\author{
Satyake Bakshi ${ }^{1}$, Sathya $A^{2}$ \\ ${ }^{1}$ Department of Systems and Computer Engineering, Carleton University, Canada \\ ${ }^{2}$ Department of Sensors and Biomedical Technology, VIT University, India
}

\begin{tabular}{lll}
\hline Article Info & ABSTRACT \\
\cline { 3 - 3 } Article history: & $\begin{array}{l}\text { AdaBoost along with HaarCascades have been well received for its accuracy } \\
\text { and perfor-mance in primarily Facial Recognition applications. However, } \\
\text { they are known to perform poorly with objects which have a different } \\
\text { Received Jan 9, 2019 }\end{array}$ & $\begin{array}{l}\text { rotational orientation or for objects whose shapes are largely variant. In this } \\
\text { paper we apply Adaptive Cascading technique to a specific derma-tological } \\
\text { application of detecting red acne which are largely shape variant outgrowths } \\
\text { Accepted Feb 28, 2019 }\end{array}$ \\
$\begin{array}{l}\text { on the skin and to identity its suitability in detection of acne. Based on the } \\
\text { outcome it would be de-clared if Viola Jones based Adaptive Boosting is } \\
\text { well suited for dermatological processing of skin diseases. }\end{array}$ \\
Accuracy metrics
\end{tabular}

Haar features

Integral image

\section{Corresponding Author:}

Satyake Bakshi,

Department of Systems and Computer Engineering,

Carleton University,

Ottawa, Ontario, Canada.

Email: satyakebakshi@cmail.carleton.ca, satyakeb@gmail.com

\section{INTRODUCTION}

The Modality of detection plays an important role in the diagnosis of most types of diseases. Artificial Intelligence and Neural networks have played an instrumental role in bridging the gap between errors in detection. Machine Learning based feature extractions have been applied to tasks like image classification and recognition of objects of same or different classes in medical images [1]. However, Machine Learning is not always useful for tasks which are trivial as it leads to higher computational costs and memory requirements of running complex simula-tions on simple models [2].

Ideally speaking Machine Learning algorithms should be usable by non-experts.But are typically not the case. There are problems of overfitting, underfit-ting, Gradient vanish-ing, Gradient exploding and countless other problems which lead to a nonsensical interpretation of the final results [3]. Hence it takes a lot of expert assumptions to design a Ma-chine Learning Algorithm.

There are countless different types of algorithms availa-ble with each drawback and advantages. To Name, a few the problems of making use of HaarFea-tures is the orientation of the image. If the orientation of the object which it has trained for has changed i.e. rotated or hidden partially behind some other object, the algorithm fails to identify the object. Algorithms like SIFT (Scale Invariant Feature Transform) corrects this by identifying unique key points from Differ-ence of Gaussian-based operations in different octaves and encodes them in vector descriptors which meet a certain sta-bility criterion. The Rotational invariance is done by finding the rotational assignment of the magnitude of the gradient. The SIFT was developed by David Lowe of UBC[4]. Other modern detection algorithms like the SSD, YOLO exists [5]. These are highly complex in nature how-ever all of them aim to reduce the computational cost of operations on Images/Videos. The SSD is also referred to as the Single Shot MultiBox Detector [6]. It uses 
multiple boxes over the image and performs $\mathrm{CNN}$ (Convolutional Neural Network) based classifica-tion operation on each box of various sizes. The best-chosen Box is decided after training in which the object resides in. The most important feature of this method is that it can utilize features across different layers i.e. "Strength in Numbers".

Problem: The Viola Jones based adaptive boosting is not suitable for detecting features having non-uniform shapes and strucutres. Despite this drawback it is still a very fast and efficient algorithm

Proposed Solution: Here we evaluate the possibility of using multiple custom handcrafted features to train the model and to evaluate the performance of the algorithm on images having uneven shape profile here in this case Red skin leisions. This could be of significant interest since cosmeticians wouldn't have to manually look for such defects on the skin to assess individual severity of these outgrowths. Instead the algorithm can be used to quantify the extent of damage on the outgrowths of the skin.

\section{RESEARCH METHOD}

\subsection{Haar Features and Adaptive Boosting}

Figure 1 Shows the haar masks.

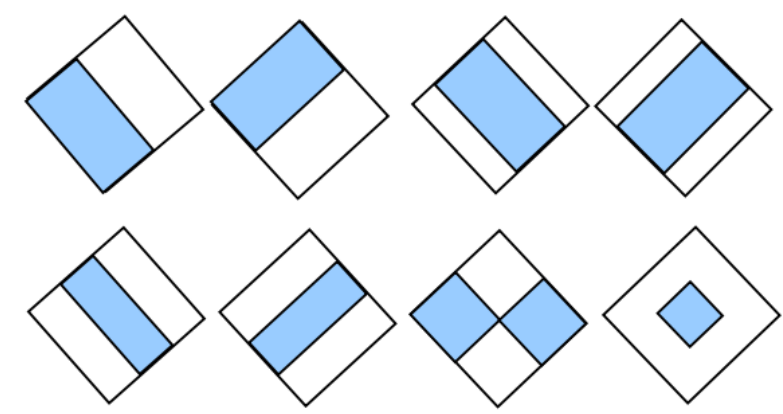

Figure 1. Shows the haar masks

Typically, there are 4 types of Haar Features i.e. Edge Features, Line Features, and Four-Square Features. These Features represent certain characteristics of an image. For example, if we are to identify a nose in the next image, we would make use of the line or edge feature. The central budging of the nose represents pixels with brighter value and the sides of the nose have pixels of low intensity. Hence depending on the type of application, the chosen haar features are applied. The Viola-Jones finds how close the intensities are with the ideal case. The positive values are averaged, and the Negative values are averaged and subtracted. To get a certain value which can be compared with how close it is to 0 or 1 .

Figure 2 Shows the application of Haar Feature on an image of identifying a nose (From Bing).

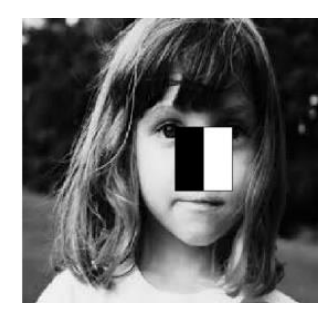

Figure 2. Shows the application of Haar Feature on an image of identifying a nose (From Bing)

Training is done by making use of positive and negative images. The positive images are of the objects which we want to detect, and the negative images are of arbitrary nature but should not have the object we are training the classifier for in the images. Typically, it is desired that there should be at least a thousand negative and 
positive images for good training. The algorithm scales down the images to $24 \mathrm{X} 24$ and the features are scaled up [7].

Adaptive Boosting is a technique employed to identify strong features using weak features [8]. The relation can be mathematically written as

$$
F(x)=\alpha f(x)+\alpha f(x) \ldots \ldots \ldots \ldots+\alpha f\left(x_{n}\right)
$$

Where $F(x)$ is the strong classifier and $f(x)$ are weak classifier $\alpha$ is the weight of that classifier.

Cascading is then performed. The main advantage of cascading is that it is very fast[9].

A sub window is taken and the features which the classifier is trained for are searched if the most important features are missing then the classifier rejects that window. It then moves with a stride of N. If the classifier finds some features relevant to the object it is trained for it looks for the second most relevant features and if it is found looks for the third until all the features are identified. If any of the feature is missing in one or more of the subsequent steps the sub-window is terminated.

Intuitive Example: If we are identifying a face the algorithm first looks for eyes and if it is present it looks for nose and if present looks for lips .etc. This continues until it finds the features defining a face.

\subsection{Creating Handcrafted $X M L$}

A third-party application has been used to custom create the cascade files. The application is designed by an algorithm Dasardh [10]. The positive images are put in the raw-data folder and the negative images are put in the negative folder. Create_list.batch file is then run. This creates a list of all the negative images with their associated file names. In the positive Folder, Object-Marker is launched which allows for custom cropping of Regions of Interest on each of the individual training images (positive). The areas of interest are cropped and saved in the form of a rectangle of coordinates (x, y, x1, y1) with the associated intensity values. This has an advantage that using object marker we can crop multiple ROI from one image. Hence results in feature augmentation i.e. increasing the number of features form a small pool of images.

Sample_Creations.batch file is then run which creates a vector file following which we use the file haar train to train the algorithm. After the cascade files are generated it is converted to XML format by making use of convert. Batch file, which creates the XML file with custom features.

The Next images would show the contents and the generation of custom cascades shown in Figures 3-10.

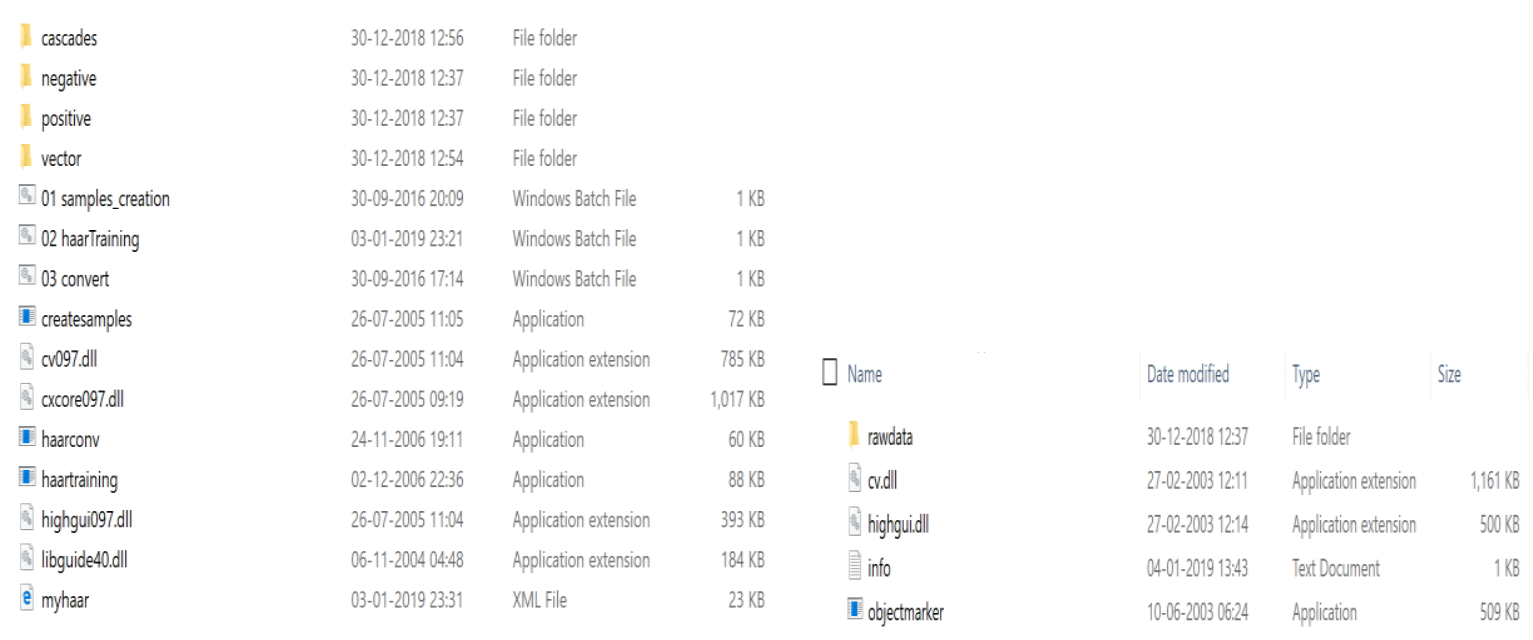

Figure 3 . The files in the third party software
Figure 4. The raw data is where positive images are put 


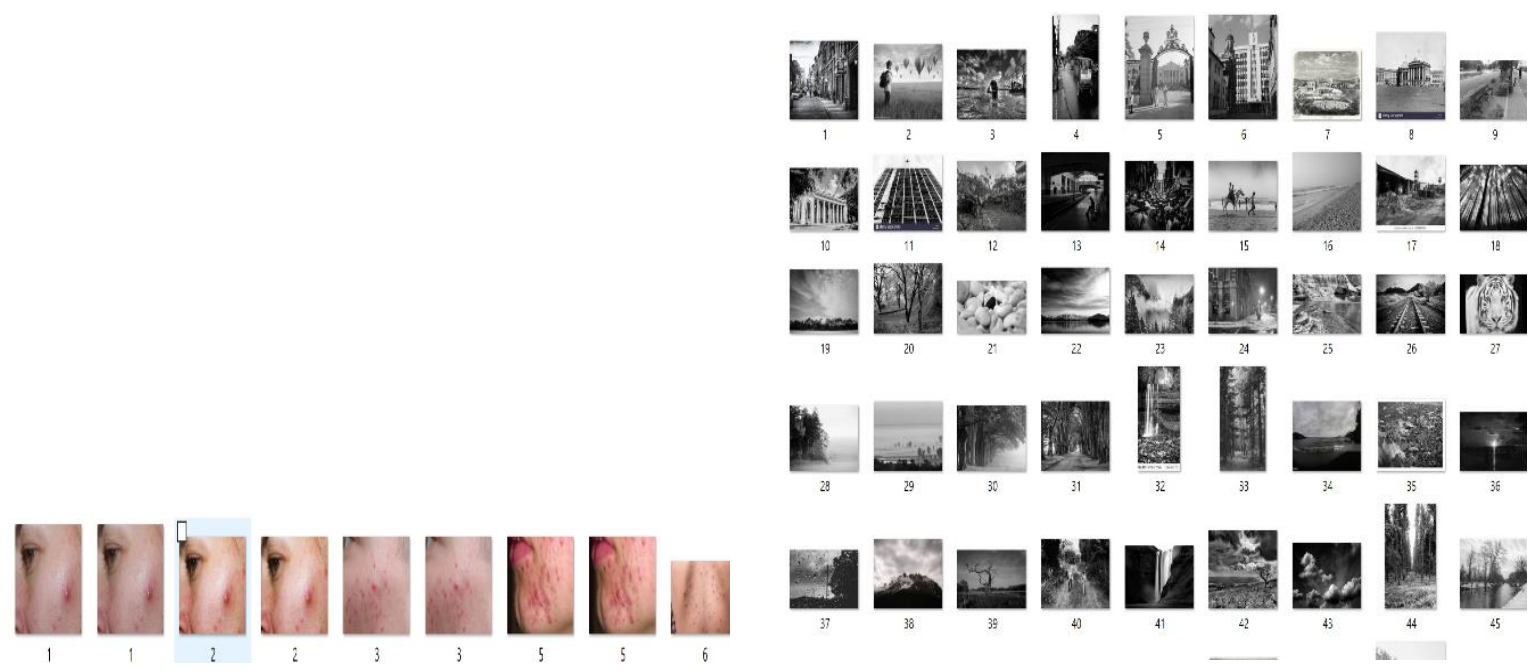

Figure 5. Positive images

Figure 6. The list of negative images

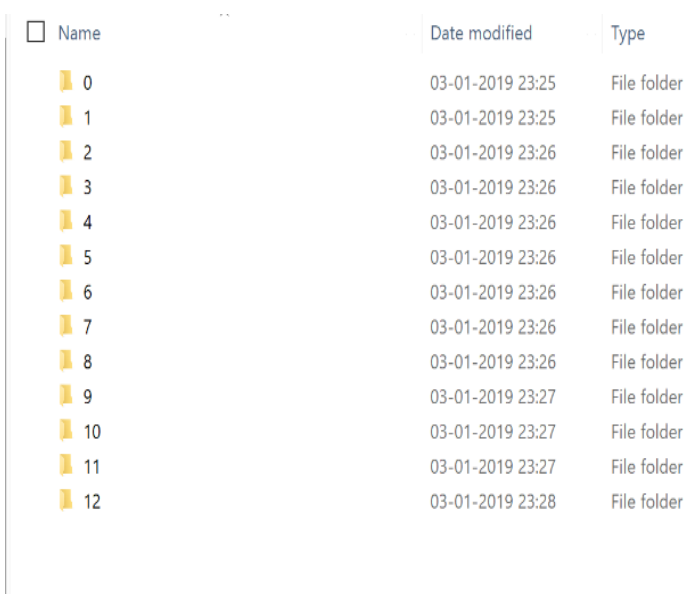

Figure 7. The generated cascade files

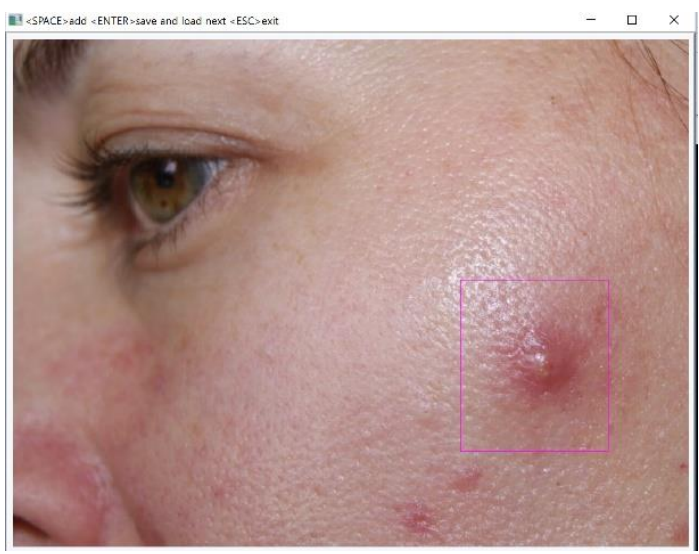

Figure 9. Handcrafting features of interest

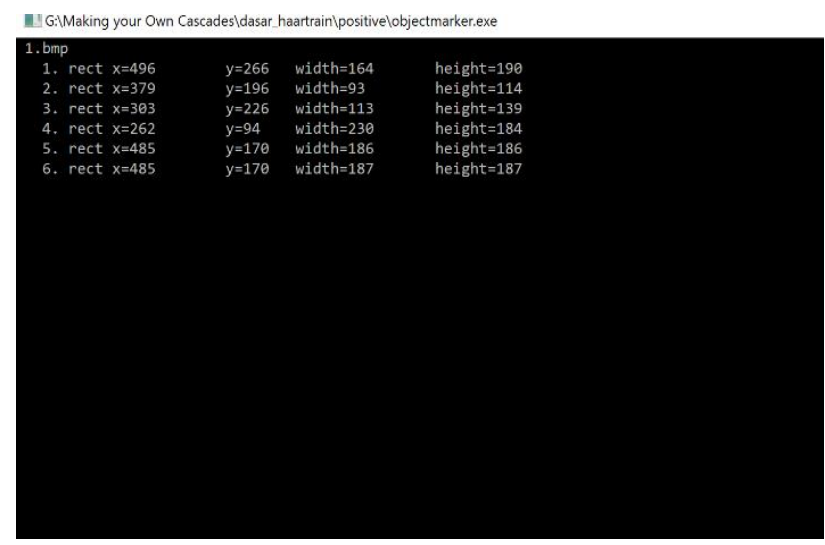

Figure 10. Values picked up from the crop 


\section{RESULTS AND DISCUSSION}

After the cascade files are generated, we implement standard AdaBoost implementation in Open $\mathrm{CV}$. Using the classic function detect MultiScale with set parameters of Scale 1.0001 and Neighborhood of 1. The Parameters were chosen which resulted in the largest number of detected bounding boxes. Figure 11(a) and Figure 11(b) shows test image and the hits and misses marked in red and blue (these have been thoroughly analyzed by repeated inspection to derive a rough estimate of the accuracy of the system.

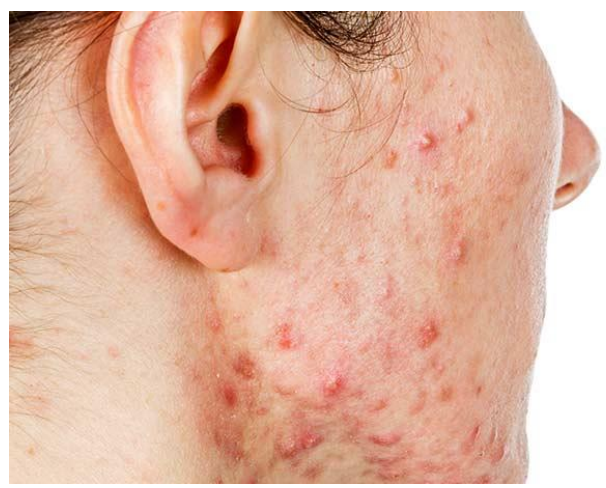

Figure 11(a). Test Image \#1 [11]
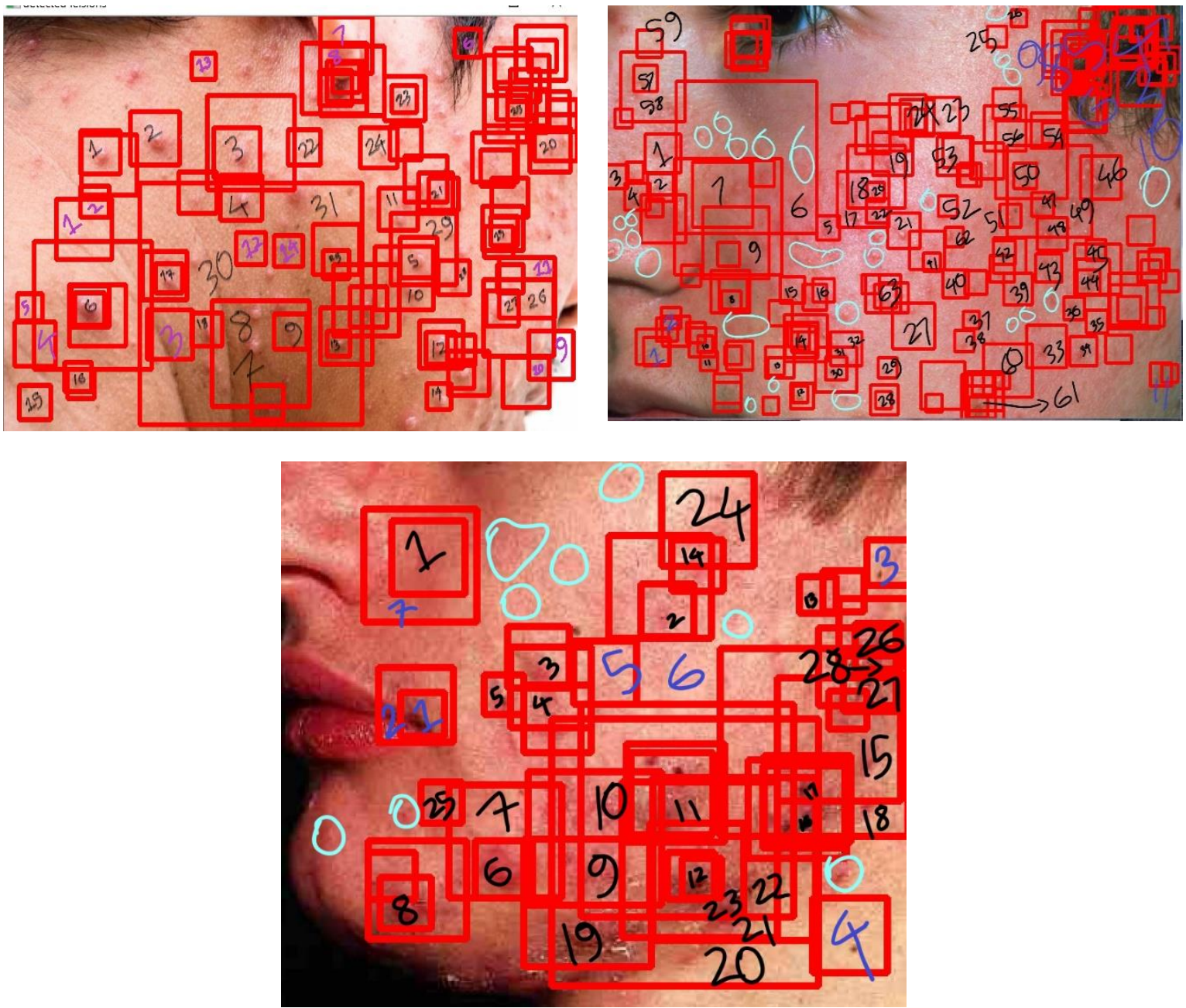

Figure 11(b). The hits and misses marked in red and blue (these have been thoroughly analyzed by repeated inspection to derive a rough estimate of the accuracy of the system 
The model yielded very good accuracy and performance with respect to sensitivity. The number of True Positive Detection outnumbers the False Negative by a lot of margins hence it can be concluded the algorithm performs sufficiently well for detecting Red Acne or for any superficial lesion detection.

Figure 12 Shows the sensitivity of the model. TP v/s FP Curve is shown in Figure 13.

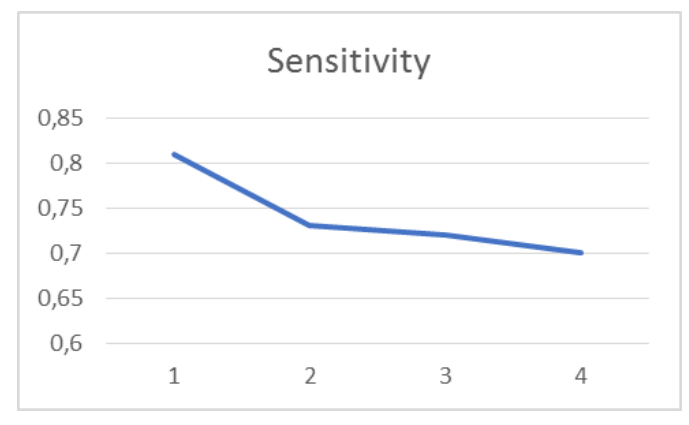

Figure 12. Sensitivity of the model

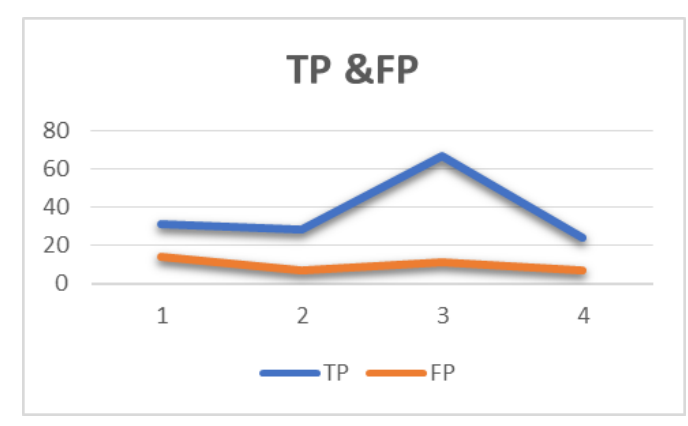

Figure 13. TP v/s FP curve

There is still an issue of multiple overlapping bounding boxes generated from the algorithm. This can be corrected by making use of NMS algorithm. Hence it has been shown that Cascading works sufficiently well for applications not only pertaining to facial recognition but also for dermatological applications.

\section{CONCLUSION}

Hence it is seen that Viola Jones based adaptive boosting method performs quite well with handcrafted features pertaining to irregular objects of interest here in this case red acnes. Henceforth it can be concluded that Viola Jones even though having a significant False positive rate the True Positives are quite accurate in most cases within the scope of this study. Hence it can be fairly assumed that this algorithm is quite efficient in highlighting important features form custom training as opposed to earlier claims of it not being able to detect uneven features.

\section{REFERENCES}

[1] R. C. Deo, "Machine learning in medicine," Circulation, 2015.

[2] M. J. Kearns, "The Computational Complexity of Machine Learning," mitpressmitedu, 1990.

[3] M. Kubat, An Introduction to Machine Learning. 2017.

[4] T. Lindeberg, "Scale Invariant Feature Transform," Scholarpedia, 2012.

[5] J. Redmon and A. Farhadi, "YOLO9000: Better, faster, stronger," in Proceedings - 30th IEEE Conference on Computer Vision and Pattern Recognition, CVPR 2017, 2017.

[6] W. Liu et al., "SSD: Single shot multibox detector," in Lecture Notes in Computer Science (including subseries Lecture Notes in Artificial Intelligence and Lecture Notes in Bioinformatics), 2016.

[7] S. Soo, "Object detection using Haar-cascade Classifier," Inst. Comput. Sci. Univ. Tartu, 2014.

[8] P. Favaro and A. Vedaldi, "AdaBoost," in Computer Vision, 2014.

[9] J. Sochman and J. Malas, "AdaBoost with totally corrective updates for fast face detection," Sixth IEEE Int. Conf. Autom. Face Gesture Recognition, 2004. Proceedings., 2004.

[10] Dassardh makes (2019-01-08).Custom Classifier. Retrieved from youtube.

[11] Google Images,Skin Leision, Date Accessed,24/01/2019 